Portland State University

PDXScholar

Electrical and Computer Engineering Faculty

Publications and Presentations

$5-2011$

\title{
Quantum Phase Estimation Using Multivalued Logic
}

Marek Perkowski

Portland State University, marek.perkowski@pdx.edu

Vamsi Parasa

Portland State University

Follow this and additional works at: https://pdxscholar.library.pdx.edu/ece_fac

Part of the Electrical and Computer Engineering Commons

Let us know how access to this document benefits you.

Citation Details

Perkowski, Marek and Parasa, Vamsi, "Quantum Phase Estimation Using Multivalued Logic" (2011). Electrical and Computer Engineering Faculty Publications and Presentations. 202.

https://pdxscholar.library.pdx.edu/ece_fac/202

This Conference Proceeding is brought to you for free and open access. It has been accepted for inclusion in Electrical and Computer Engineering Faculty Publications and Presentations by an authorized administrator of PDXScholar. Please contact us if we can make this document more accessible: pdxscholar@pdx.edu. 


\title{
Quantum Phase
}

\section{Estimation using}

Multivalued Logic

\author{
Vamsi Parasa \\ Marek Perkowski
}

Department of Electrical and Computer Engineering, Portland State University

ISMVL 2011, 23-25 May 2011, Tuusula, Finland 


\section{Agenda}

- Importance of Quantum Phase Estimation (QPE)

- QPE using binary logic

- QPE using MVL

- Performance Requirements

- Salient features

- Conclusion

ISMVL 2011, 23-25 May 2011, Tuusula, Finland 


\section{Abstract}

- We generalize the Quantum Phase Estimation algorithm to MVL logic.

- We show the quantum circuits for QPE using qudits.

- We derive the performance requirements of the QPE to achieve high probability of success.

- We show how this leads to logarithmic decrease in the number of qudits and exponential decrease in error probability of the QPE algorithm as the value of the radix $d$ increases. 


\section{Introduction}

- QPE - one of the most important quantum subroutines, used in :

-1) Shor's Algorithm

-2) Abrams and Lloyd Algorithm

- (Simulating quantum systems)

- Calculation of Molecular Ground State Energies

- 3) Quantum Counting (for Grover Search)

-4) Fourier Transform on arbitrary $Z_{p}$

ISMVL 2011, 23-25 May 2011, Tuusula, Finland 


\section{Quantum phase estimation (QPE)}

Let $|u\rangle$ be the eigenstate of a unitary operator $U$ with an eigenvalue $e^{2 \pi i \varphi_{u}}$, where the value of the phase $\varphi_{u}$ is unknown. The goal of the QPE algorithm is to determine the best approximation to the phase $\varphi_{u}$.

ISMVL 2011, 23-25 May 2011, Tuusula, Finland 


\section{QPE - Formal Definition}

- More formally, if we have $U|u\rangle=e^{2 \pi i \varphi_{u}}|u\rangle$

Let $\varphi_{u} \approx \frac{\tilde{\varphi}_{u}}{d^{t}}=0 . \tilde{\varphi}_{1} \tilde{\varphi}_{2} \tilde{\varphi}_{3} \ldots . \tilde{\varphi}_{t-1} \tilde{\varphi}_{t}$ be the best $t$ 'dit' approximation to $\varphi_{u}$

- This implies,

$$
\frac{\tilde{\varphi}_{u}}{d^{t}}=\tilde{\varphi}_{1} d^{-1}+\tilde{\varphi}_{2} d^{-2}+. . \tilde{\varphi}_{t-1} d^{-(t-1)}+\tilde{\varphi}_{t} d^{-t}
$$

for each $\tilde{\varphi}_{i} \in[0, d-1]$.

- Thus the goal of the QPE algorithm is to find the value of $\tilde{\varphi}_{u}$ which gives the best estimate of the original phase $\varphi_{u}$ 


\section{QPE Algorithm- Binary logic case}

We first review the binary case:

Let $\varphi_{u} \approx \frac{\tilde{\varphi}_{u}}{2^{t}}=\tilde{\varphi}_{1} 2^{-1}+\tilde{\varphi}_{2} 2^{-2}+. . \tilde{\varphi}_{t-1} 2^{-(t-1)}+\tilde{\varphi}_{t} 2^{-t}$ be the best $t$ bit approximation to $\varphi_{u}$. In short, it is represented as $\varphi_{u} \approx 0 . \tilde{\varphi}_{1} \tilde{\varphi}_{2} \tilde{\varphi}_{3} \ldots \tilde{\varphi}_{t-1} \tilde{\varphi}_{t}$

\section{Schematic for the QPE Algorithm}

Eigenvector of $U$
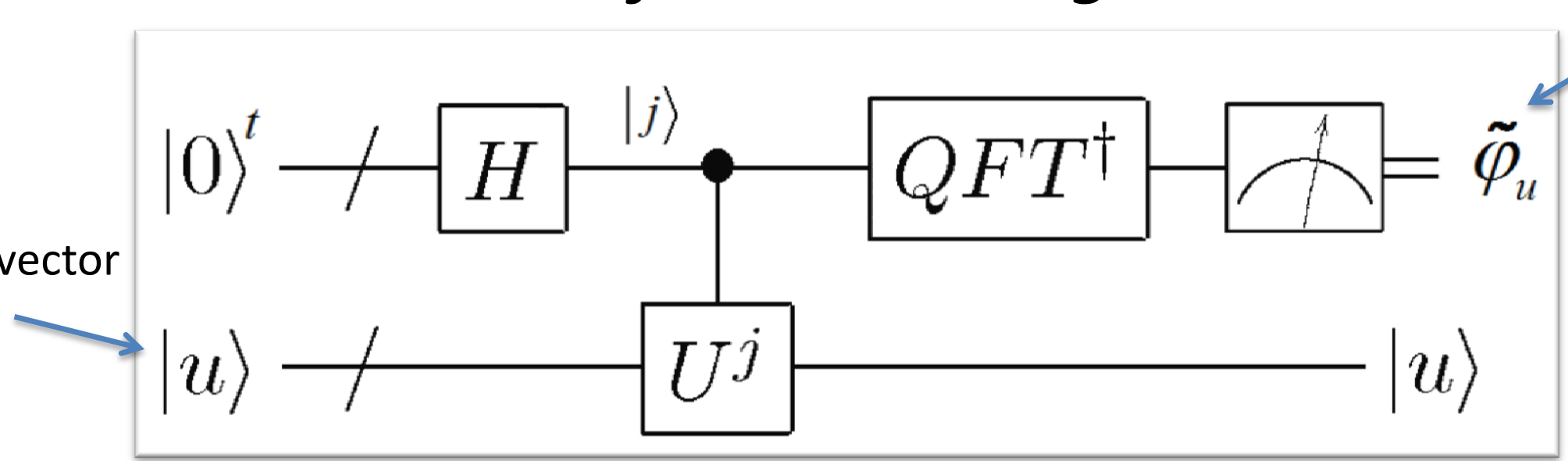

Measure phase in $\mathrm{t}$ qubits 
QPE : step 1 - Initialization, Binary

\section{logic case}

$|0\rangle^{t}$ be the tensor product of $t$ qubits each in the state $|0\rangle$

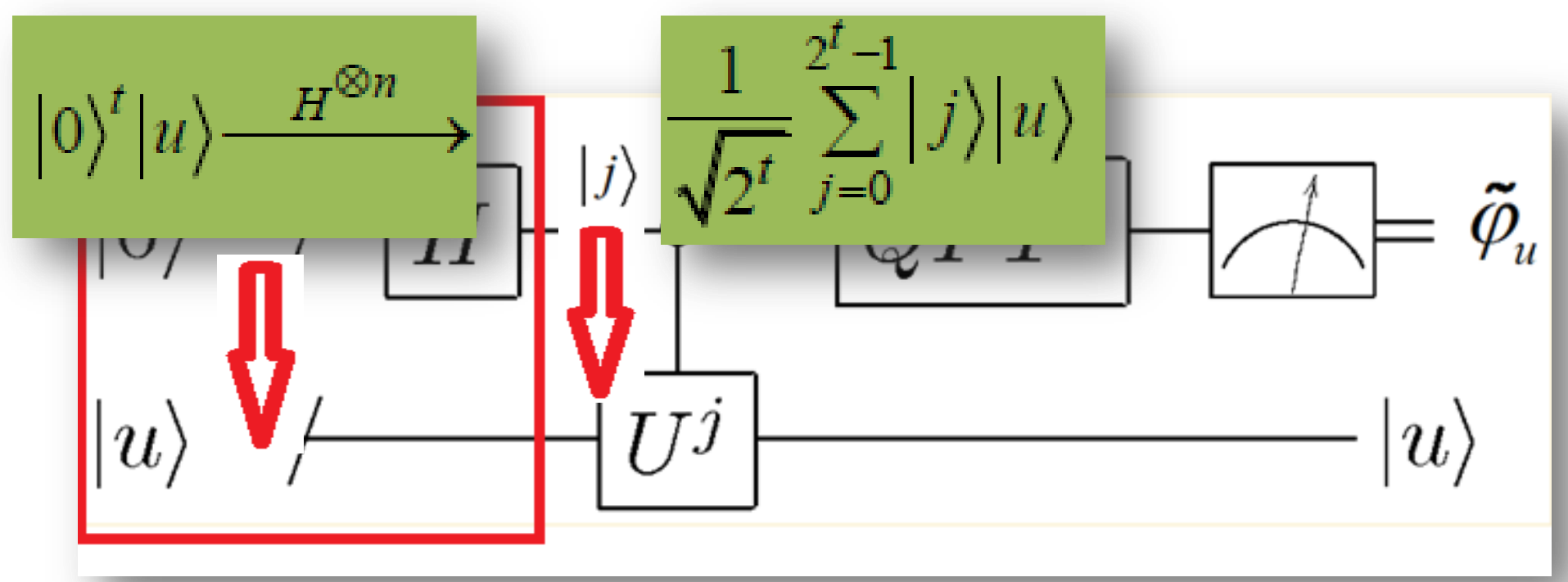

ISMVL 2011, 23-25 May 2011, Tuusula, Finland 


\section{QPE : step 2-Apply the operator U}

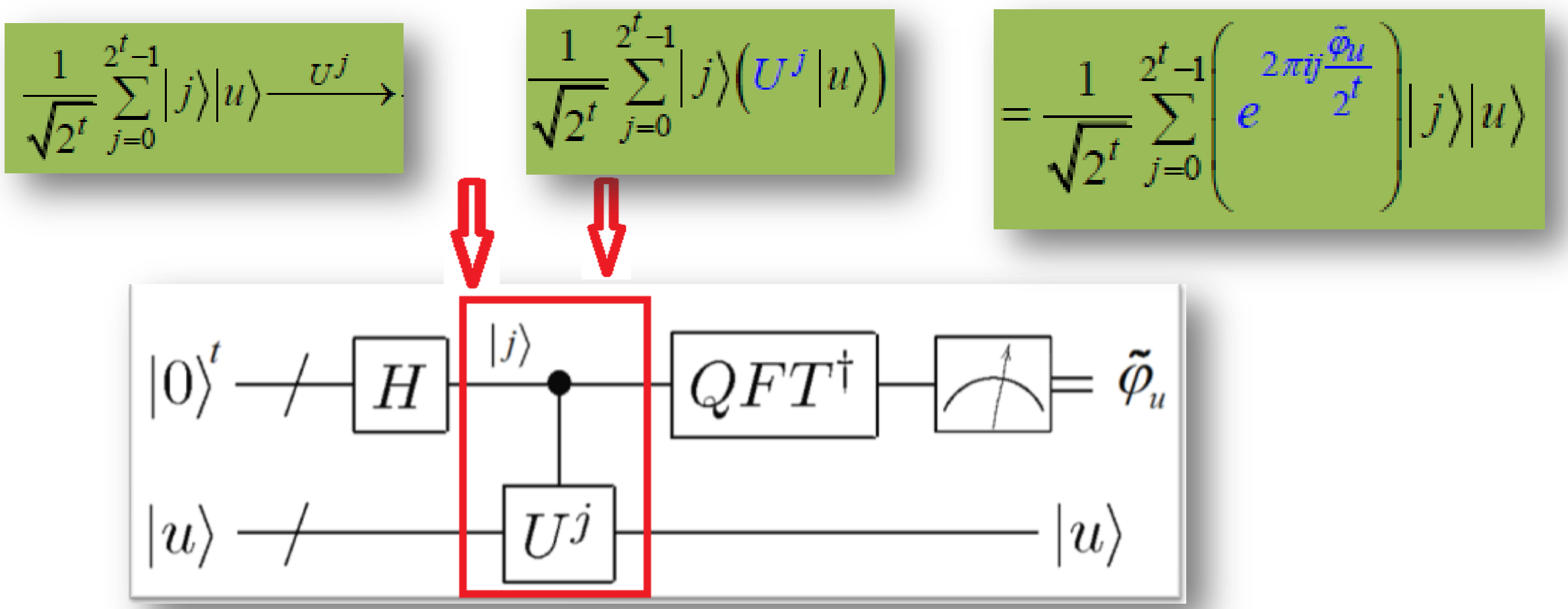

$\hat{U} U^{j}$ denotes the controlled unitary operator $\mathrm{U}$
controlled on the state vector $|j\rangle$.

Binary logic case

$$
\left(U^{j}|u\rangle\right)=\left(e^{2 \pi i \varphi_{u}}\right)^{j}|u\rangle=\left(e^{2 \pi i \frac{\tilde{\varphi}_{u}}{2^{t}}}\right)^{j}|u\rangle
$$




\section{QPE : step 3 - Apply Inverse QFT}

Binary logic case

$$
\frac{1}{\sqrt{2^{t}}} \sum_{j=0}^{2^{t}-1}\left(e^{2 \pi j j} \frac{\tilde{\varphi}_{u}}{2^{t}}\right)|j\rangle|u\rangle \stackrel{Q F T^{\dagger}}{\longrightarrow}\left|\tilde{\varphi}_{u}\right\rangle|u\rangle
$$

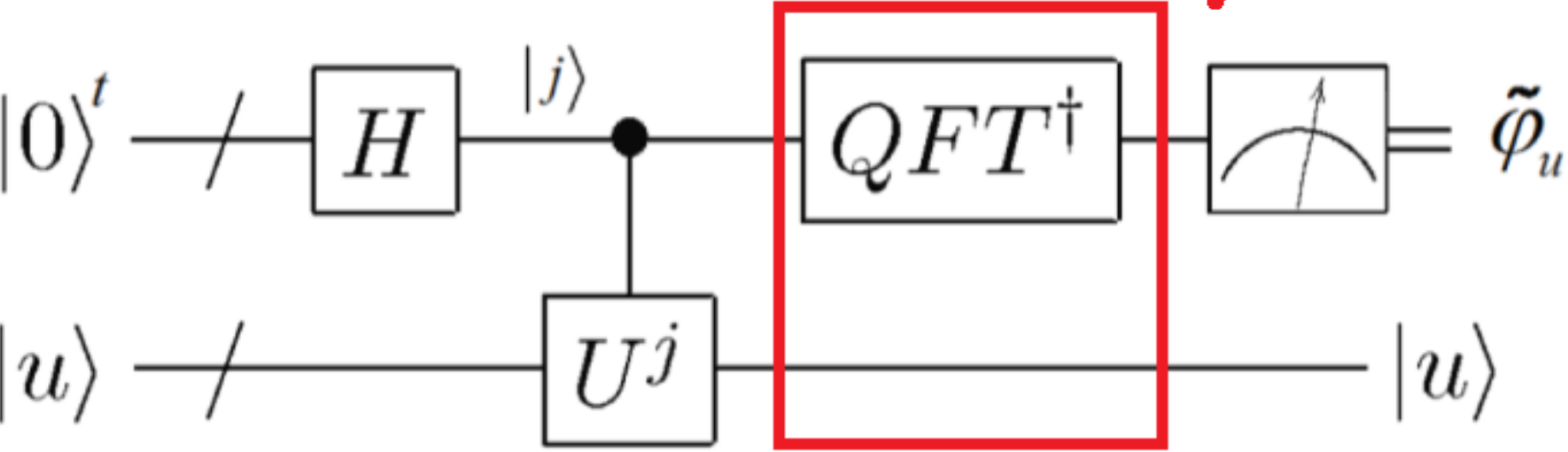

$$
\text { QFT Definition: }|j\rangle \rightarrow \frac{1}{\sqrt{2^{n}}} \sum_{k=0}^{2^{n}-1} e^{\frac{2 \pi i j k}{2^{n}}}|k\rangle
$$




\section{QPE : step 4 - Measurement}

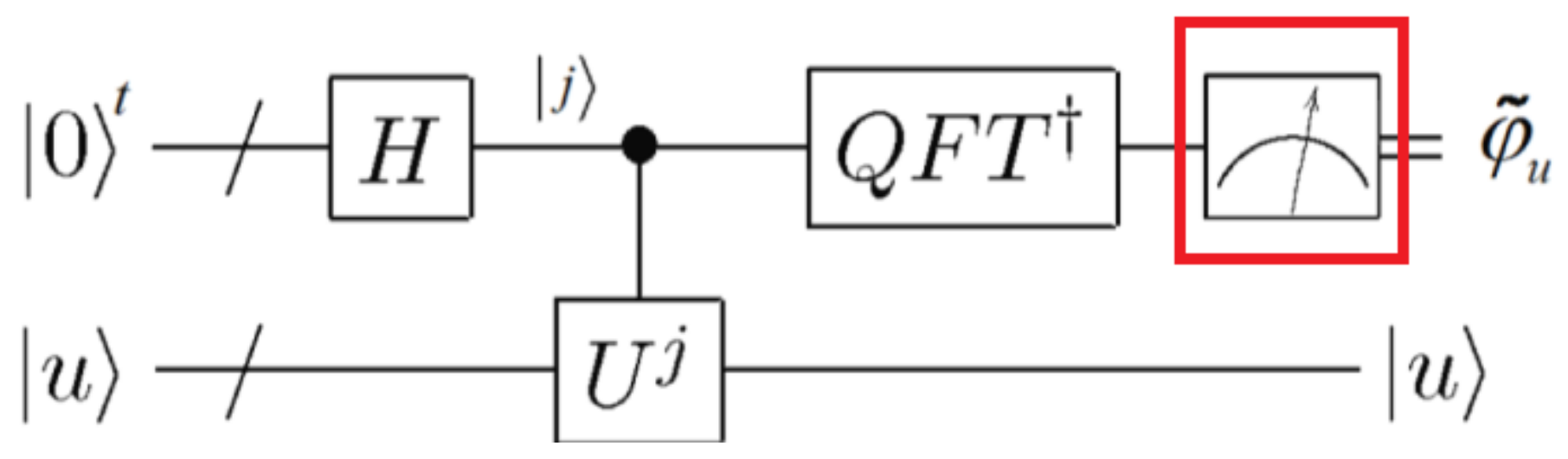

Binary logic case

- If the phase $?_{\mathrm{u}}$ is an exact binary fraction, we measure the estimate of the phase $?_{u}$ with probability of 1 .

- If not, then we measure the estimate of the phase with a very high probability close to 1 . 


\section{Quantum circuit for $\boldsymbol{u}^{j}$}

$$
\begin{aligned}
& \text { If } j=j_{1} 2^{t-1}+j_{2} 2^{t-2}+\ldots j_{k} 2^{t-k} \ldots+j_{t-1} 2^{1}+j_{t} 2^{0} . \\
& U^{j}=U^{j_{1} 2^{t-1}} \times U^{j_{2} 2^{t-2}} \times \ldots U^{j_{k} 2^{t-k}} \ldots \times U^{j_{t-1} 2^{1}} \times U^{j_{t} 2^{0}}
\end{aligned}
$$

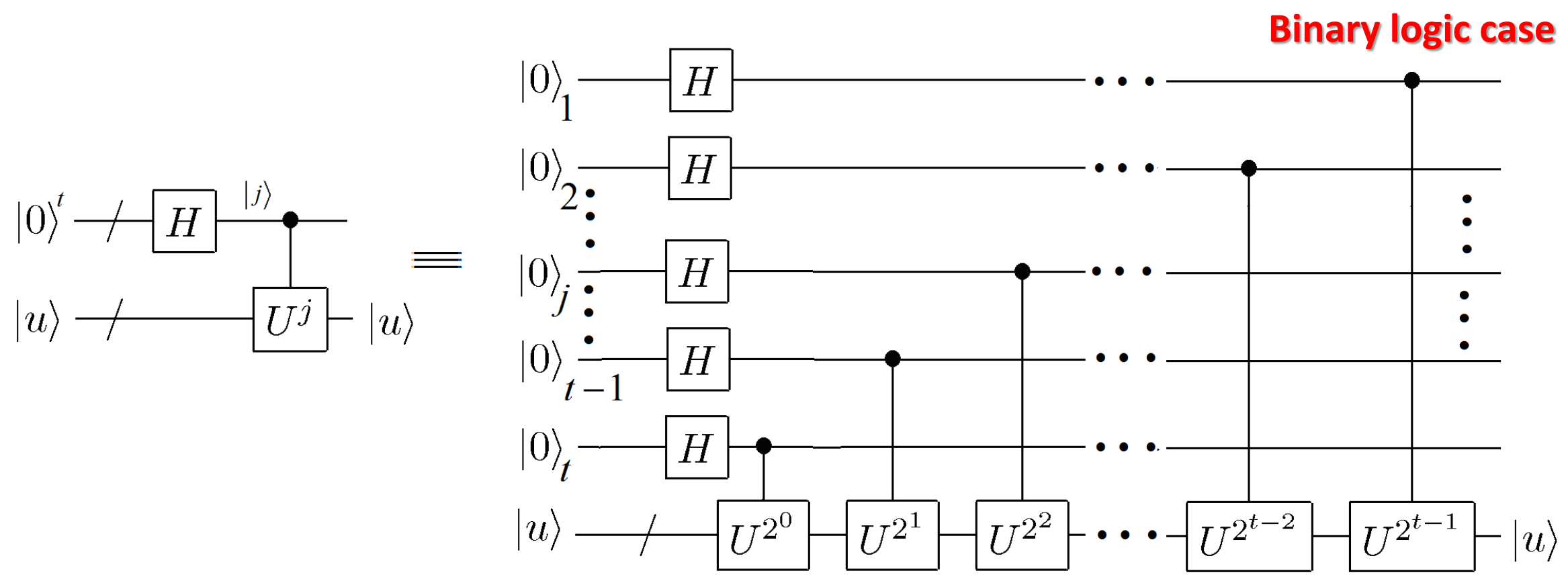

The circuit for QFT is well known and hence not discussed. 


\section{QPE - Generalization to MVL}

MV logic case

- We represent the phase $\varphi_{\imath \text { as a }} d$-ary fraction given by

We have $t$ qudits for phase

$$
\varphi_{u} \approx \frac{\tilde{\varphi}_{u}}{d^{t}}=0 . \tilde{\varphi}_{1} \tilde{\varphi}_{2} \tilde{\varphi}_{3} \ldots . \tilde{\varphi}_{t-1} \tilde{\varphi}_{t}
$$

$$
\frac{\tilde{\varphi}_{u}}{d^{t}}=\tilde{\varphi}_{1} d^{-1}+\tilde{\varphi}_{2} d^{-2}+. . \tilde{\varphi}_{t-1} d^{-(t-1)}+\tilde{\varphi}_{t} d^{-t}
$$

Now we have qudits not qubits

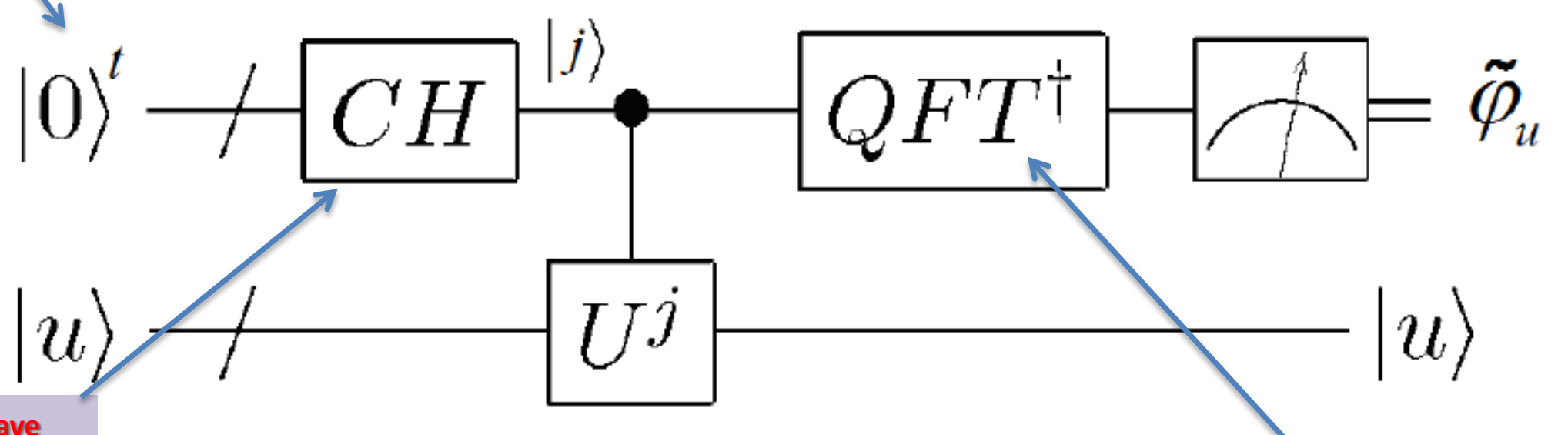

Now we have arbitrary Chrestenson instead Hadamard
Schematic for QPE using Qudits Now we Inverse QFT on base d, not base 2 


\section{Some definitions af and chrestenson}

MV logic case

- The Multivalued logic QFT on $\boldsymbol{n}$ qudits is defined as :

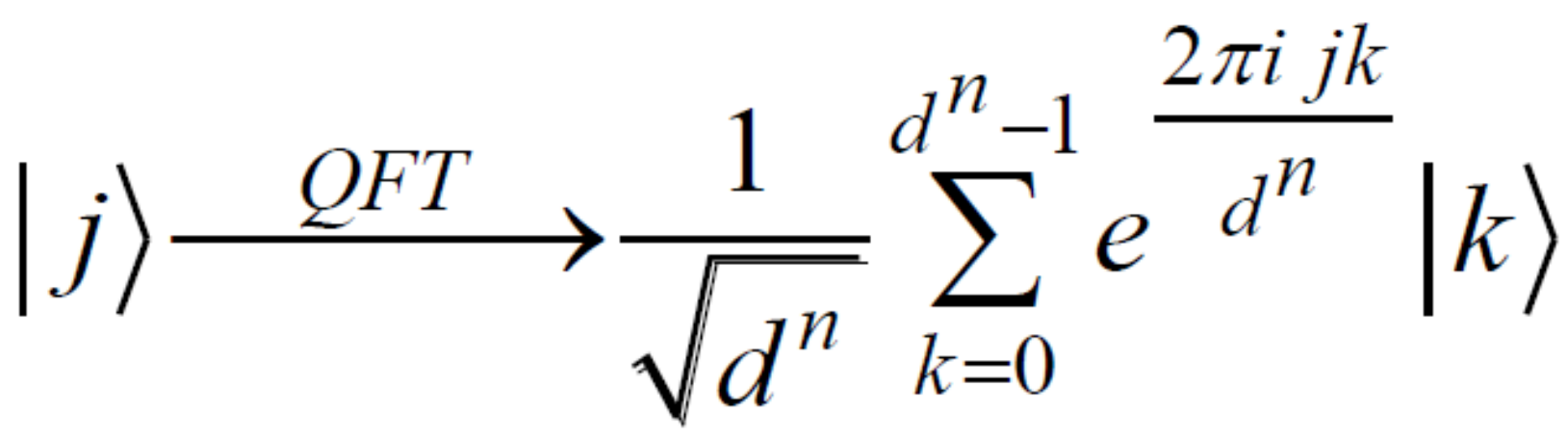

- The action of a Chrestenson $(\mathrm{CH})$ gate on a single qudit is defined as:

$$
C H|x\rangle \rightarrow \frac{1}{\sqrt{d}} \sum_{y \in[0, d-1]} e^{\frac{2 \pi i x y}{d}}|y\rangle
$$

For $d=2$, the $\mathrm{CH}$ gate reduces to Hadamard gate 


\section{MVL QPE : Step 1 - Initialization}

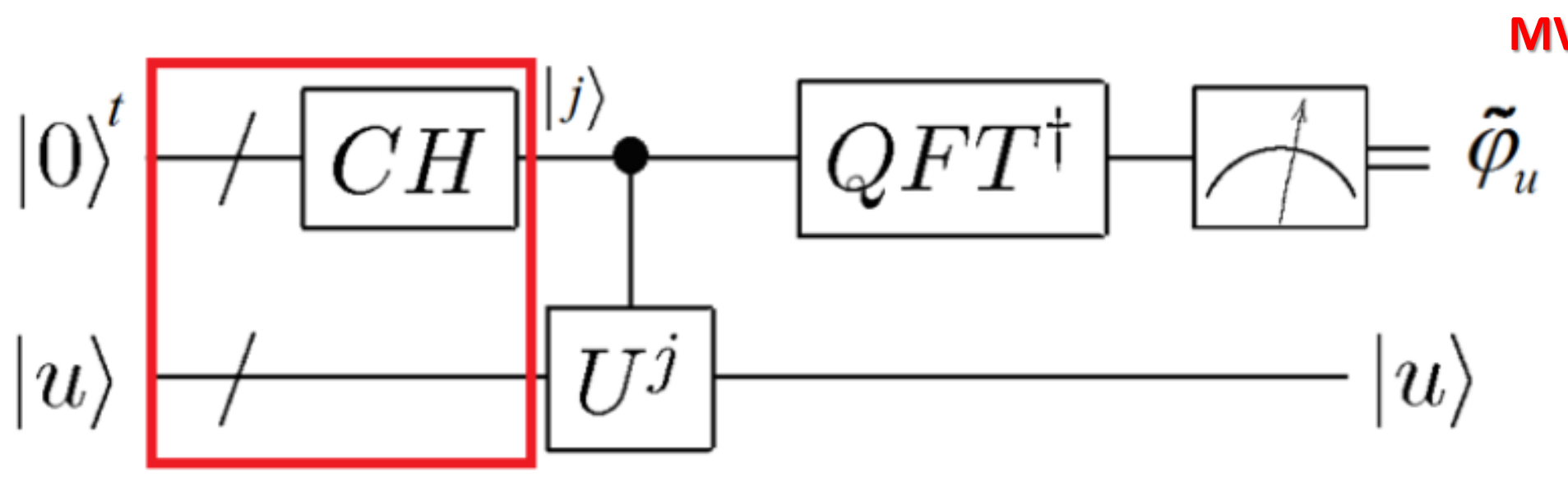

MV logic case

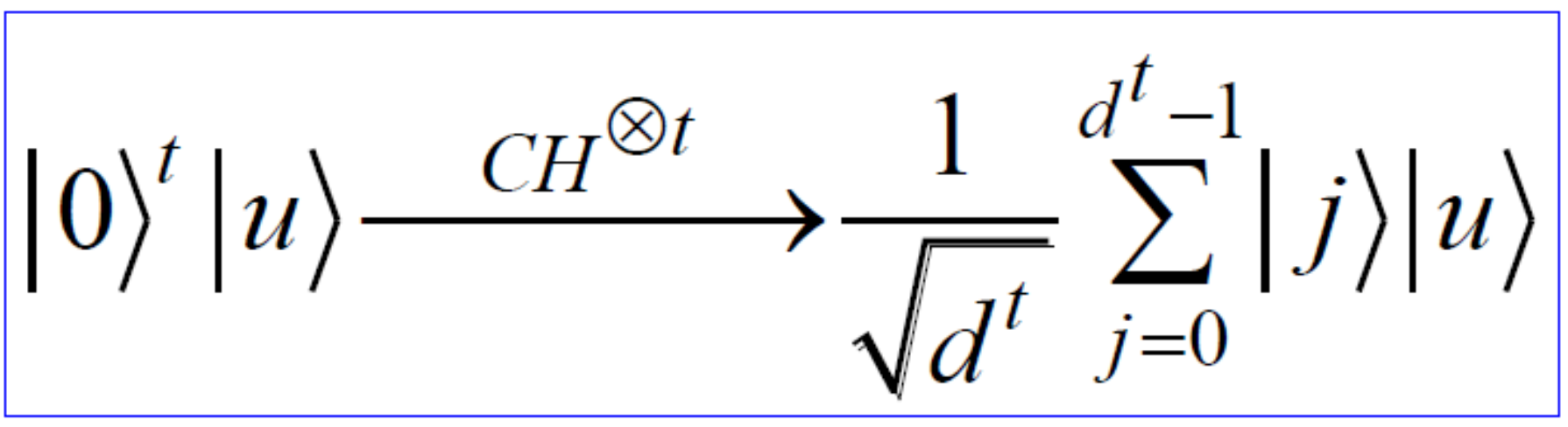

$$
\left(C H|0\rangle \rightarrow \frac{1}{\sqrt{d}} \sum_{j \in[0, d-1]}|j\rangle\right)
$$




$$
\frac{1}{\sqrt{d^{t}}} \sum_{j=0}^{d^{t}-1}|j\rangle|u\rangle \stackrel{U^{j}}{\longrightarrow} \frac{1}{\sqrt{d^{t}}} \sum_{j=0}^{d^{t}-1}|j\rangle\left(U^{j}|u\rangle\right)
$$

MV logic case

$$
=\frac{1}{\sqrt{d^{t}}} \sum_{j=0}^{d^{t}-1}|j\rangle\left(e^{2 \pi i \varphi_{u}}\right)^{j}|u\rangle=\frac{1}{\sqrt{d^{t}}} \sum_{j=0}^{d^{t}-1}\left(e^{2 \pi i j \frac{\tilde{\varphi}_{u}}{d^{t}}}\right)|j\rangle|u\rangle
$$

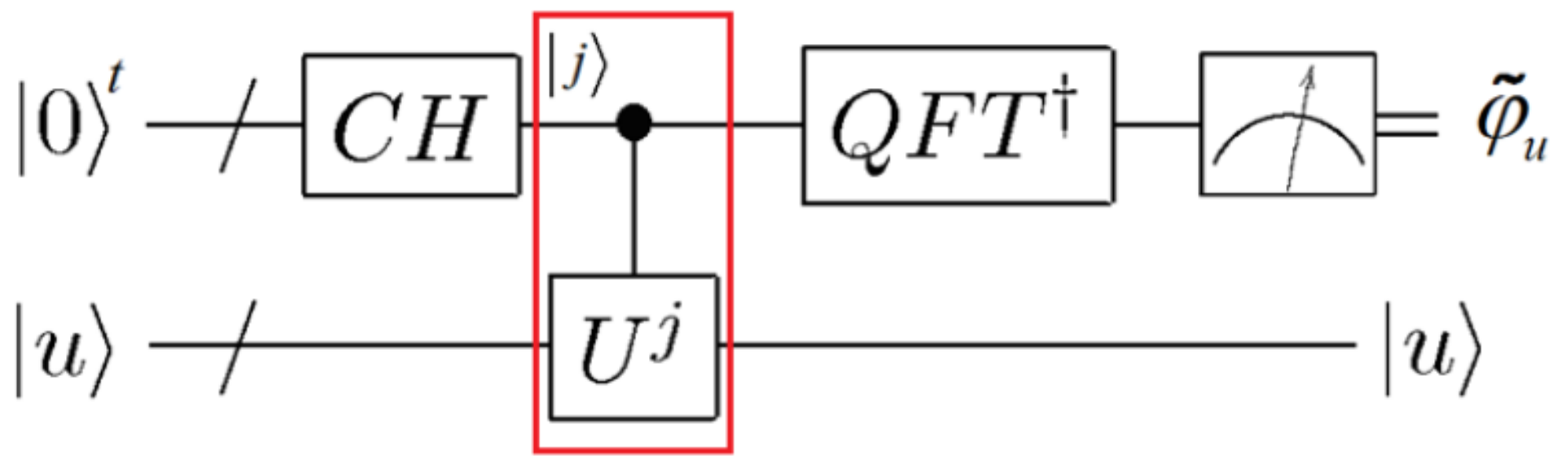

ISMVL 2011, 23-25 May 2011, Tuusula, Finland 


\section{MVL QPE: Step 3 - apply iqft}

MV logic case

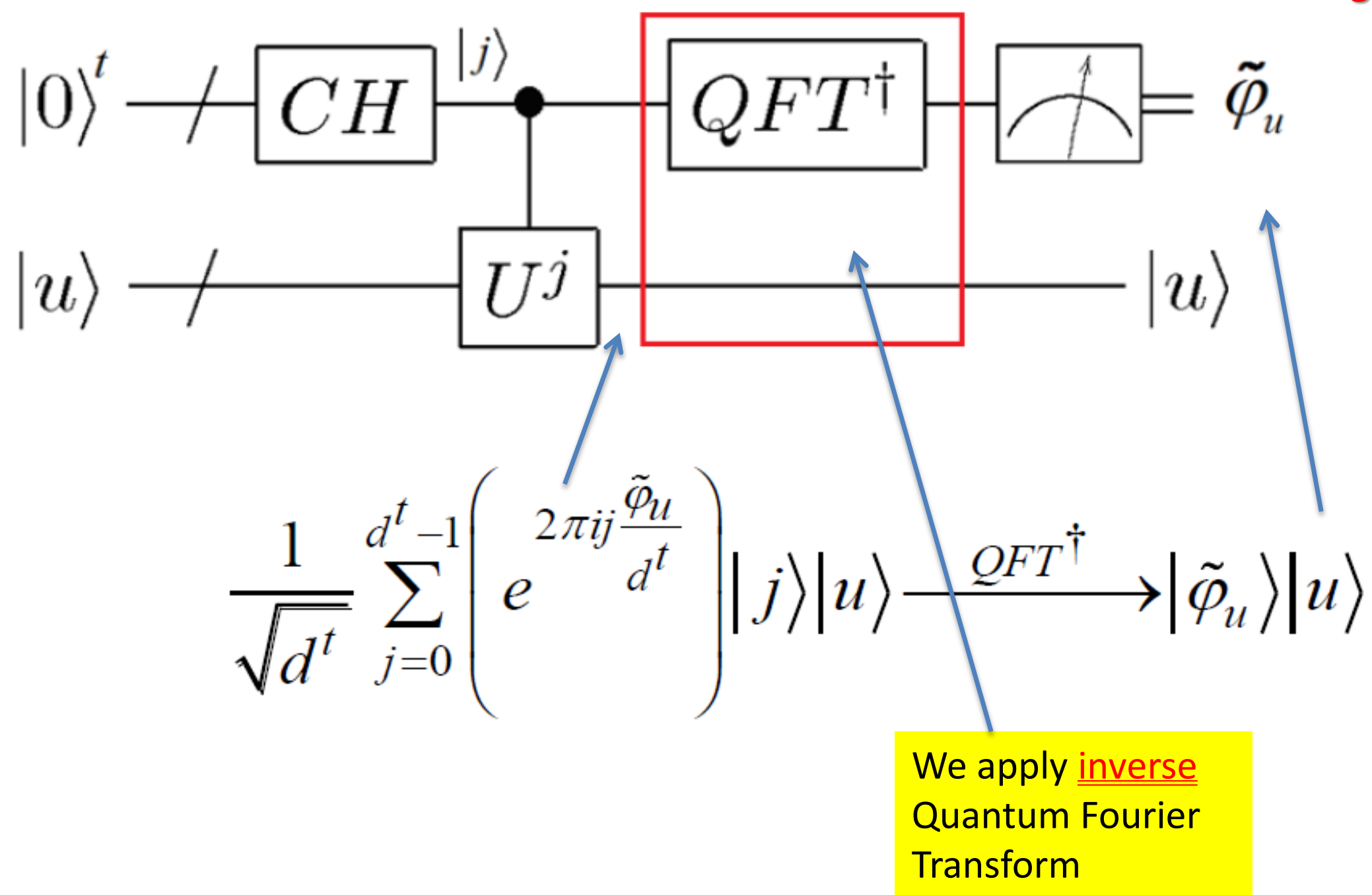

ISMVL 2011, 23-25 May, Tuusula, Finland 


\section{MVL QPE: Step 4 -- measurement}

MV logic case

- After making a measurement on the first qudit register, we now get $\tilde{\varphi}_{u}$ which is an estimate of the phase $\varphi_{u}$

- If $\varphi_{u}$ is not an exact $d$-ary fraction then we can only measure the phase with a high success probability close to 1 but not exactly 1 . 


\section{Quantum circuit for $u^{j}$}

Let $|j\rangle=\otimes_{k=1}^{t}\left|J_{k}\right\rangle \quad \Rightarrow U^{j}=U^{\left(\sum_{k=1}^{t} J_{k} d^{t-k}\right)}=\prod_{k=1}^{t} U^{J_{k} d^{t-k}}$

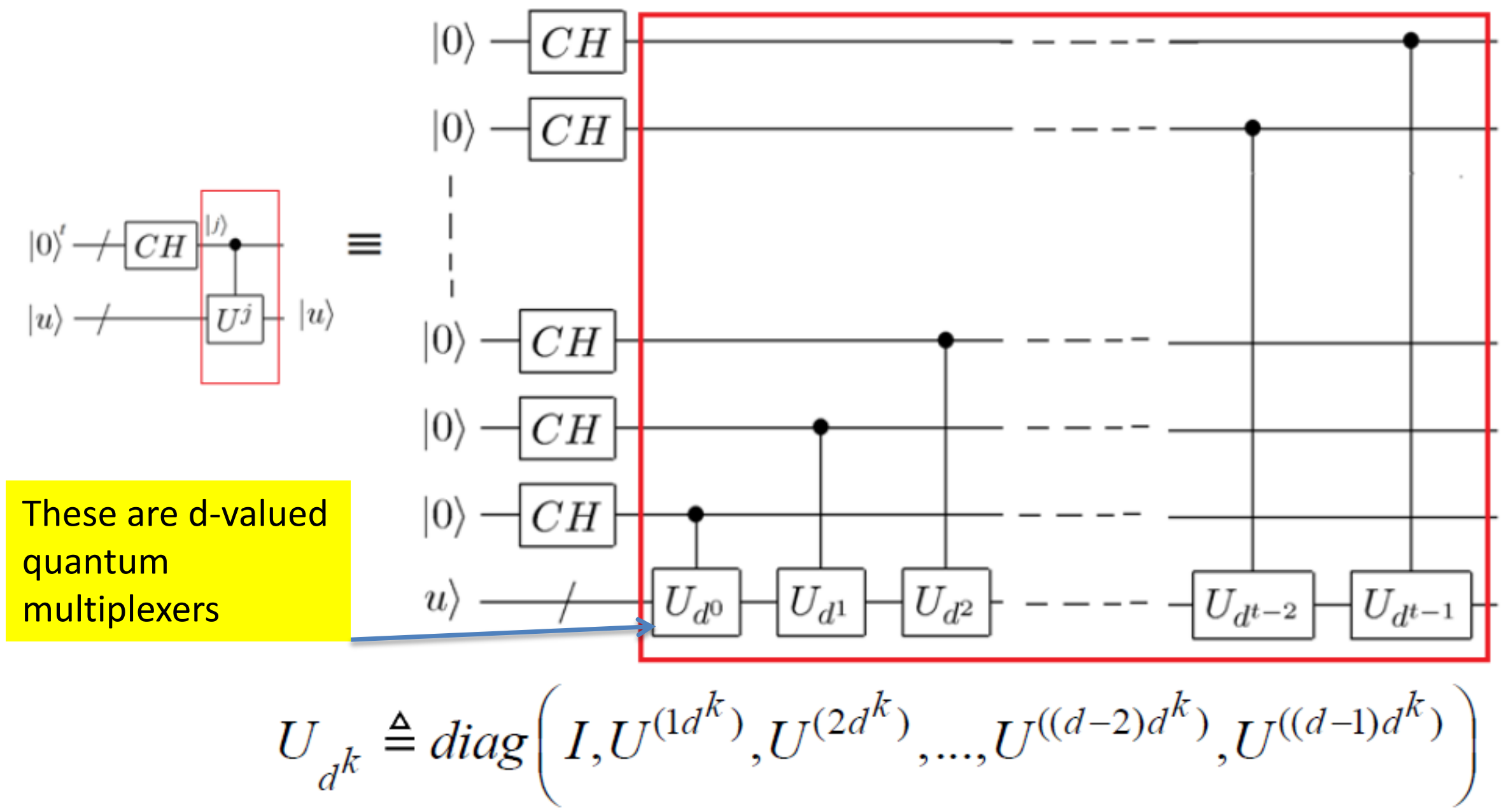




\section{D-valued quantum multiplexers}

Case $d=3$

control

Target (date)

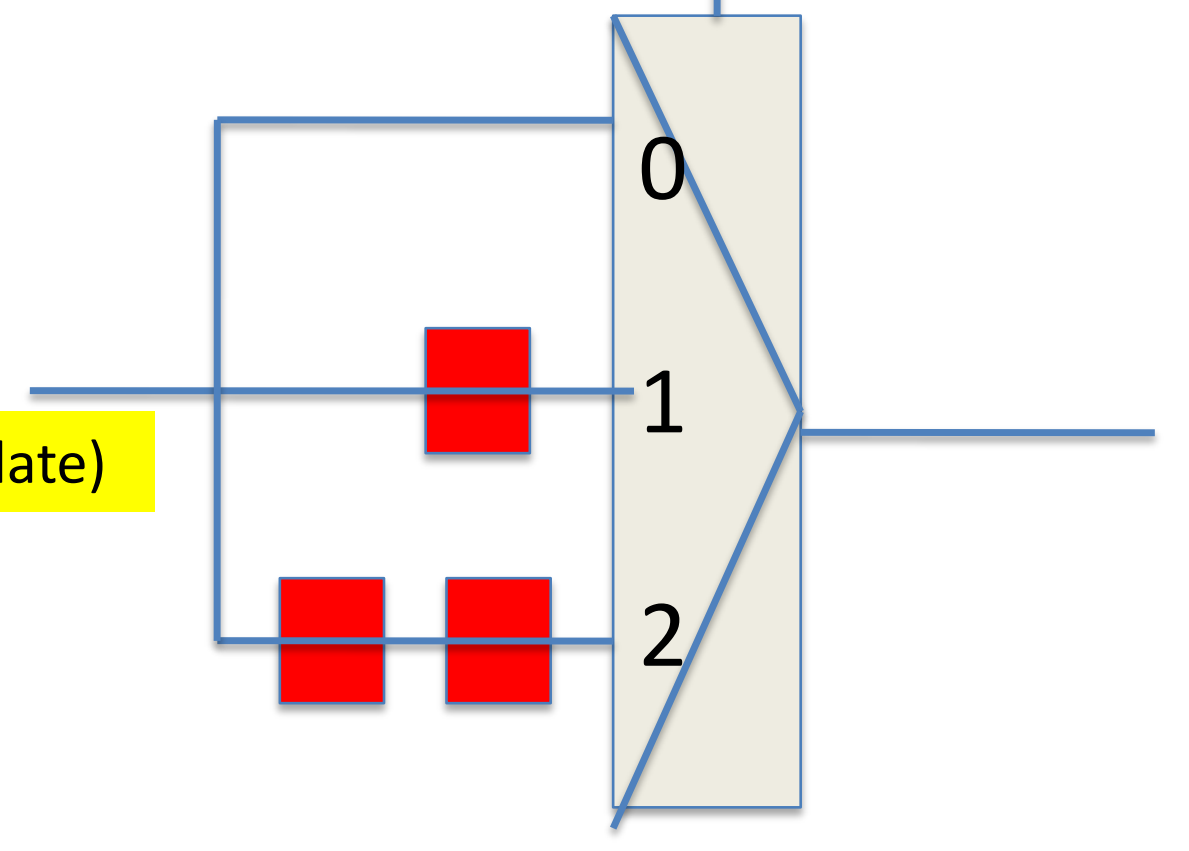




\section{QUANTUM Circuit}

- IQFT can be implemented with a complexity of $\mathrm{O}(\mathrm{n} \log n)$ but the expensive part is implementing higher order powers of $U$.

- This determines the complexity of the circuit.

ISMVL 2011, 23-25 May, Tuusula, Finland 


\section{QPE - Performance}
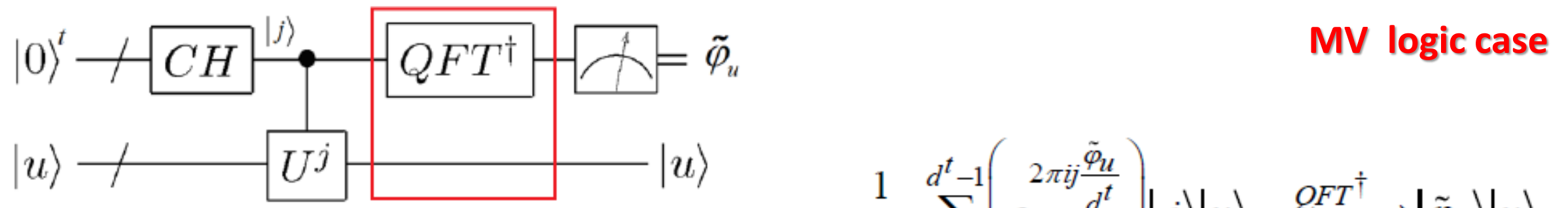

$$
\frac{1}{\sqrt{d^{t}}} \sum_{j=0}^{d^{t}-1}\left(e^{2 \pi i j \frac{\tilde{\varphi}_{u}}{d^{t}}}\right)|j\rangle|u\rangle \stackrel{Q F T^{\dagger}}{\longrightarrow}\left|\tilde{\varphi}_{u}\right\rangle|u\rangle
$$

If the phase $\boldsymbol{\varphi}_{\tilde{\varphi}_{\boldsymbol{u}}}$ is an exact d-ary fraction i.e.

$\varphi_{u}=\frac{\tilde{\varphi}_{u}}{d^{t}}$ then after QFT ... QPE algorithm gives correct answer with probability of 1 .

What if it is not an exact faction ?

$$
\text { i.e. } \quad \varphi_{u}=\frac{\tilde{\varphi}_{u}}{d^{t}}+\delta \quad\left(0 \leq \delta<d^{-t}\right)
$$

ISMVL 2011, 23-25 May, Tuusula, Finland 


\section{QPE Performance}

binary

- It can be shown that, in the general case, when the phase is not an exact fraction, QPE succeeds with minimum probability of $\frac{8}{\pi^{2}}=81.5 \%$

-What can we do to increase this success probability very close to 1 ?

- Will MVL help in this aspect? YES 


\section{QPE Performance: PHASE $\neq$ d-ary fraction}

- Let's analyze what happens if the phase is not an exact fraction. Applying the QFT gives a new superposition state

$$
\frac{1}{\sqrt{d^{t}}} \sum_{j=0}^{d^{t}-1}\left(e^{2 \pi i j \frac{\tilde{\varphi}_{u}}{d^{t}}}\right)|j\rangle \stackrel{Q F T^{\dagger}}{\longrightarrow} \sum_{l=0}^{d^{t}-1} \alpha_{l}|l\rangle
$$

It is not hard to show that, the probability of measuring an $l$ given by $P(l)$ is

$$
P(l)=\left|\alpha_{l}\right|^{2}=\left|\frac{1}{d^{t}} \sum_{j=0}^{d^{t}-1} \exp \left(2 \pi i \frac{\left(\varphi_{u}-j\right)}{d^{t}} l\right)\right|^{2}
$$




\section{QPE Performance}

- Using the fact that $\varphi_{u}=\frac{\tilde{\varphi}_{u}}{d^{t}}+\delta \quad\left(0 \leq \delta<d^{-t}\right)$

We get $P(l)=\left|\alpha_{l}\right|^{2}=\left|\frac{1}{d^{t}}\left(\frac{1-e^{2 \pi i\left(d^{t} \delta+\left(\tilde{\varphi}_{u}-l\right)\right)}}{1-e^{2 \pi i\left(\delta+\frac{\tilde{\varphi}_{u}-l}{d^{t}}\right)}}\right)\right|^{2}$

MV logic case

- Thus after measurement, we get some value $l$ with the probability given above. i.e. this implies $\varphi_{u}=\frac{l}{d^{t}}$

- If $l$ is close to $\tilde{\varphi}_{l l}$ then we can say QPE succeeded else QPE fails.

- How close is close? 


\section{QPE : Success Probability lower bound}

- It is easy to show that the probability that QPE returns either $l$ or $l+l$ such that $l \leq \tilde{\varphi}_{u} \leq l+1$ is $\frac{8}{\pi^{2}}=81.5 \%$ as

$$
P(l)=\left|\alpha_{l}\right|^{2}=\left|\frac{1}{d^{t}} \frac{1-e^{2 \pi i\left(d^{t} \varphi_{u}-l\right)}}{1-e^{2 \pi i\left(\varphi_{u}-\frac{l}{d^{t}}\right)}}\right| \geq \frac{4}{\pi^{2}}
$$

- Although encouraging, the lower bound is not good enough.

- We need SUCCESS PROBABILITY close to 1.

- How to define SUCCESS PROBABILITY? 


\section{Success probability = 1 - failure}

\section{probability}

MV logic case

- Suppose we have a $t$ dit approximation to the phase

$$
\varphi_{u} \approx \frac{\tilde{\varphi}_{u}}{d^{t}}=0 . \tilde{\varphi}_{1} \tilde{\varphi}_{2} \tilde{\varphi}_{3} \ldots . \tilde{\varphi}_{t-1} \tilde{\varphi}_{t}
$$

- If we are only interested in a precision of only upto $n$ dits

$$
\text { i.e. } \varphi_{u}=\frac{\tilde{\varphi}_{u}}{d^{t}}+\delta \quad\left(0 \leq \delta<d^{-n}\right)
$$

- then as long as QPE returns some li.e. $\varphi_{u}=\frac{l}{d^{t}}$ such that the above condition is satisfied, we have a success.

- The error $e$ is

$$
\begin{aligned}
& e=l-\tilde{\varphi}_{u}=\delta d^{t} \text { but }\left(0 \leq \delta<d^{-n}\right) \\
& \Rightarrow e<d^{t-n}
\end{aligned}
$$




\section{Failure probability}

- We define the failure probability as

$$
\varepsilon=p\left(\left|l-\tilde{\varphi}_{u}\right|>e\right)=\sum_{l=0, l \in\left[\tilde{\varphi}_{\varphi_{u}}-e, \tilde{\varphi}_{u}+e\right]}^{d^{t}-1}\left|\alpha_{l}\right|^{2} \leq \frac{1}{2(e-1)}
$$

- The failure probability has a lower bound and hence the success probability has an upper bound.

$$
\begin{gathered}
p(\text { Success })=1-\varepsilon>1-\frac{1}{2(e-1)} \\
\text { where } e=d^{t-n}-1
\end{gathered}
$$




\section{Success probability: REQUIREMENTS}

- Thus to achieve phase estimation with a success probability of $1-\varepsilon$ with precision/accuracy up to $n$ dits, we need to use a system with $t$ dits. The value of $t$ is given by

$$
t=n+p=n+\log _{d}\left(2+\frac{1}{2 \varepsilon}\right)
$$

- Now we show quantitative results in some graphs 


\section{How MVL HELPS}

- Failure probability decreases exponentially with increase in radix $d$ of the logic used

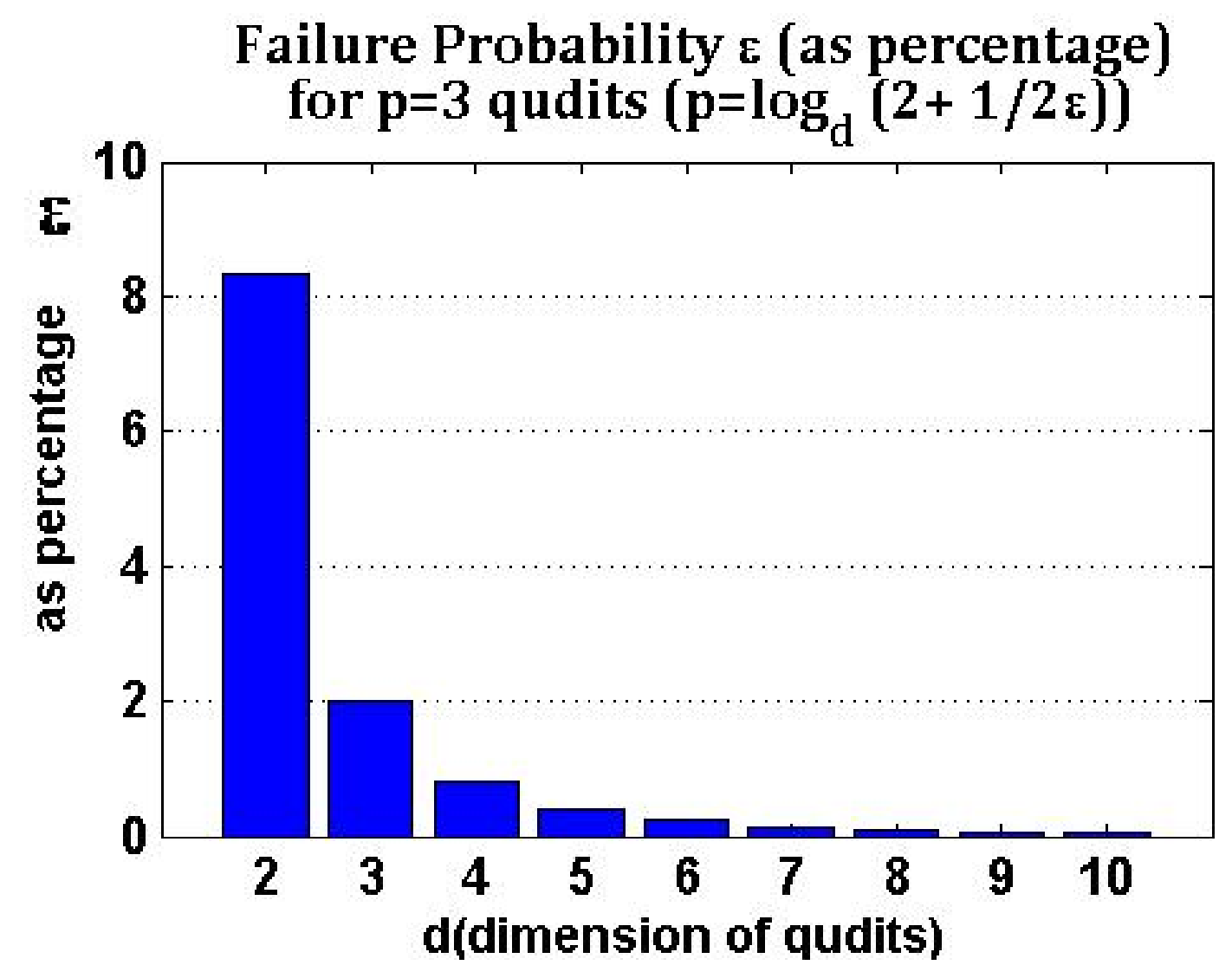




\section{Less number of qudits for a given precision}

These are the requirements for a real world problem of calculating molecular energies

Number of qudits required (t) vs dimension of qudits (d) to obtain a precision of upto 5 decimal digits with a success probability of $98 \%$

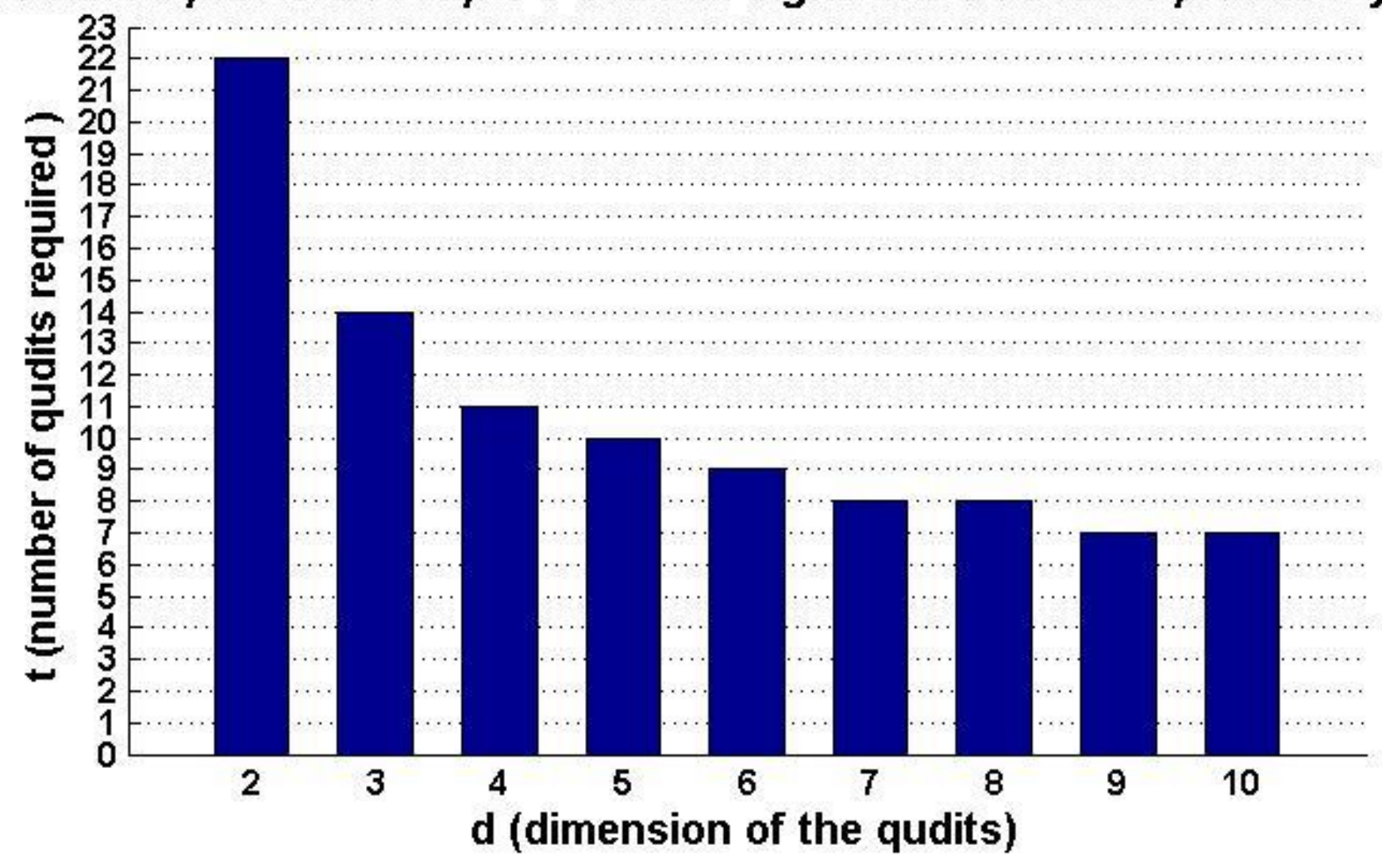




\section{More RESULTS}

NUMBER OF QUDITS REQUIRED FOR QPE ALGORITHM

\begin{tabular}{|c|c|c|c|c|c|c|}
\hline $\begin{array}{c}\text { Precision in } \\
\text { decimal digits }\end{array}$ & $\begin{array}{c}\text { Success } \\
\text { probability }\end{array}$ & $d=2$ & $d=3$ & $d=4$ & $d=5$ & $d=6$ \\
\hline 5 & $99.5 \%$ & 24 & 15 & 12 & 11 & 10 \\
\hline 4 & $98 \%$ & 19 & 12 & 10 & 9 & 8 \\
\hline 4 & $95 \%$ & 18 & 12 & 9 & 8 & 7 \\
\hline 4 & $85 \%$ & 17 & 11 & 9 & 8 & 7 \\
\hline 3 & $98 \%$ & 17 & 11 & 9 & 8 & 7 \\
\hline 2 & $99 \%$ & 14 & 9 & 7 & 6 & 6 \\
\hline 2 & $90 \%$ & 11 & 7 & 6 & 5 & 5 \\
\hline
\end{tabular}

ISMVL 2011, 23-25 May, Tuusula, Finland 


\section{Conclusions}

- Quantum Phase Estimation has many applications in Quantum Computing

- MVL is very helpful for Quantum Phase Estimation

- Using MVL causes exponential decrease in the failure probability for a given precision of phase required.

- Using MVL results in signification reduction in the number of qudits required as radix $d$ increases

ISMVL 2011, 23-25 May 2011, Tuusula, Finland 


\section{Conclusions 2}

- The method creates high power unitary matrices $\mathrm{U}^{\mathrm{k}}$ of the original Matrix $\mathrm{U}$ for which eigenvector $\mid \mathrm{u}>$ we want to find phase.

- We cannot design these matrices as powers. This would be extremely wasteful

- We have to calculate these matrices and decompose them to gates

- New type of quantum logic synthesis problem: not permutative U, not arbitrary U, there are other problems like that, we found

- This research problem has been not solved in literature even in case of binary unitary matrices $\mathrm{U}$

ISMVL 2011, 23-25 May 2011, Tuusula, Finland 